\title{
Situación de los pacientes infectados por COVID-19: un análisis para la ciudad de Bogotá (Colombia) discriminado por localidades
}

\author{
Situation of patients infected by covid-19: an analysis for the city of Bogotá discriminated by \\ locality
}

\author{
MENDOZA, Valentina ${ }^{1}$ \\ MENDOZA, Angel D. ${ }^{2}$ \\ FUENTES, Héctor J. ${ }^{3}$
}

\begin{abstract}
Resumen
En el presente estudio, se propone estimar la probabilidad que tiene una persona en Bogotá de fallecer al contraer el virus SARS-COV-2. Esta probabilidad es estimada a partir de modelos econométricos probabilísticos, para este caso a partir del Modelo Logit. Se proponen diferentes variables sociales y económicas, que pueden incidir de manera directa en las personas que residen en las diferentes localidades de la ciudad. En el estudio se evidencia que la población más afectada y de mayor probabilidad de contagiarse es la de más escasos recursos y que vive en condiciones de vulnerabilidad. Palabras clave: COVID - 19, empresas, patrones socioeconómicos, probabilidad.
\end{abstract}

\begin{abstract}
It is proposed to estimate the probability of a person in Bogotá dying when contracting the SARS-COV2 virus. This probability is estimated from probabilistic econometric models, in this case, from the Logit Model. Different social and economic variables are proposed, which can directly impact the people who reside in the different localities of the city. The study shows that the population most affected and most likely to be infected is the one with the fewest resources and lives in vulnerable conditions.

key words: COVID-19, Companies, socioeconomic patterns, probability.
\end{abstract}

\section{Introducción}

La COVID-19 es una enfermedad que demostró la fragilidad humana y ratificó su naturaleza como ser social que depende de la colectividad y la comunidad. Esta enfermedad fue notificada por primera vez en diciembre de 2019 en Wuhan (China), es catalogada como nuevo coronavirus debido a que ya se tenía un registro de esta familia de virus, que pueden producir desde un resfriado ordinario hasta enfermedades más graves con tasas de mortalidad bastante altas y este fue el último encontrado de esta familia, apodado formalmente como COVID-

\footnotetext{
${ }^{1}$ Estudiante de Ingeniería Catastral y Geodesia, Facultad de Ingeniería, Universidad Distrital Francisco José de Caldas Bogotá-Colombia, correo: vmendozam@correo.udistrital.edu.co

2 Estudiante de Ingeniería Catastral y Geodesia, Facultad de Ingeniería, Universidad Distrital Francisco José de Caldas Bogotá-Colombia, correo: admendozas@correo.udistrital.edu.co

${ }^{3}$ Docente Titular. Bogotá. Universidad Distrital Francisco José de Cladas. hjfuentesl@udistrital.edu.co
} 
19. Según datos oficiales a agosto de 2020, en el mundo se tenían más de 40 millones de casos confirmados de coronavirus y en Colombia, según el ministerio de salud, para la misma fecha aproximadamente una cifra cercana a los 800 mil casos de los cuales cerca de 700 mil ya están recuperados, un poco menos de 100 mil están activos, al igual que aproximadamente 24 mil personas habían fallecido (3.2\%). ${ }^{4}$

Como era de esperarse, el virus ha azotado de manera más drástica a la capital colombiana, ya que con sus 7.181.569 de habitantes según el último censo del Departamento Administrativo Nacional de Estadística (DANE), el 31,9 \% de los casos reportados en Colombia de Covid-19, se encuentran en Bogotá D.C. En la ciudad, se han presentado cerca de 280 mil casos confirmados de los cuales $51,4 \%$ son mujeres y la mayor concentración de casos, de acuerdo con la edad, está entre los 20 a 49 años con un peso porcentual de $60,8 \%$, según los datos del Observatorio de Salud de Bogotá (Saludata-Bogotá, 2020). La capital colombiana está dividida oficialmente en 20 localidades, para las cuales en el siguiente estudio se busca analizar, por medio de un modelo econométrico, las variables que han incidido en el aumento de la población de contagiados que ha logrado recuperarse y, principalmente, en la que ha fallecido lo cual ha traído como consecuencia, no solo una gran pérdida de vidas, sino que ha sido acompañada con incrementos sustanciales del desempleo debido al cierre de establecimientos comerciales y a la desaceleración económica que representó el confinamiento decretado por el gobierno nacional. En este orden de ideas, este artículo busca mostrar a partir de métodos cuantitativos, las probabilidades y determinantes que inciden en el fallecimiento de una persona contagiada en la capital del país, asociándola a variables de carácter social y espacial que como es sabido, no tienen la misma incidencia dependiendo del género, la edad, la actividad económica que desarrolla y sector en el que habita.

\section{Marco referencial}

La econometría es una disciplina que paulatinamente ha ganado una amplia participación en el análisis de fenómenos epidemiológicos, debido a que en sí misma, ésta se puede considerar una herramienta de análisis social que por medio de modelos matemáticos y estadísticos resulta bastante útil para el estudio de diversas investigaciones que, no solo se reducen al campo de las ciencias exactas. La COVID -19 ha demostrado tener un alcance alto en la vida de las personas, debido a esto, todos los estudios que se pueden realizar desde cualquier ámbito son vitales en la búsqueda de mayor información acerca de esta pandemia mundial declarada por la Organización Mundial de la Salud hacia marzo del año 2020 (WHO. 2020).

En este contexto y siguiendo a (Gujarati \& Porter, 2010, p. 541) si se quiere medir el impacto que unos determinantes pueden tener en una variable respuesta de carácter categórico "los modelos de regresión de respuesta cualitativa cada vez son más comunes en diversas áreas de las ciencias sociales y la investigación médica" debido a que estos permiten trabajar con una variable dependiente que puede tomar solamente dos valores dependiendo de una condición, 1 si la cumple y 0 en otro caso.

De manera específica para esta investigación, esta variable de estudio corresponde a aquellos infectados reportados como recuperados o fallecidos a los cuales se le construye una ecuación econométrica tipo Logit, en donde la variable dependiente se asocia a los contagiados que se encuentran fallecidos y recuperados.

Normalmente en los modelos econométricos se trabaja con las propiedades y aplicaciones del modelo de regresión múltiple, así, en este caso, se incorpora información cualitativa de variables explicativas mediante el uso de variables binarias. En los modelos de regresión múltiple hasta ahora, la variable dependiente obtiene un

${ }^{4}$ Datos tomados el 30 de Agosto de 2020, para mayor información consultar: https://sig.sispro.gov.co/SituacionCovid/ 
significado cuantitativo, de este modo se aplicará variables cualitativas para explicar eventos que requieran el análisis con estas variables.

De la misma manera se tiene que la característica principal de este modelo es que sigue una función de probabilidad logística tal que:

$$
\operatorname{Prob}(Y=1)=\frac{1}{1+e^{-Z_{i}}}=\frac{e^{Z_{i}}}{1+e^{Z_{i}}}
$$

donde

$$
Z_{i}=\left(\beta_{1}+\beta_{2} X_{2}+\beta_{3} X_{3}+\cdots \ldots \beta_{k} X_{k}\right)
$$

a su vez

$$
\operatorname{Prob}(Y=0)=\frac{1}{1+e^{Z_{i}}}
$$

por lo tanto

$$
\frac{P_{i}}{\left(1-P_{i}\right)}=\frac{1+e^{Z_{i}}}{1+e^{-Z_{i}}}=e^{Z_{i}}
$$

aplicando logaritmos para linealizar Zi se tiene

$$
\ln \left(\frac{P_{i}}{1-P_{i}}\right)=Z_{i}=\beta_{1}+\beta_{2} X_{2}+\beta_{3} X_{3}+\ldots \ldots \beta_{k} X_{k}
$$

La ecuación anterior es lo que se conoce como un modelo logístico en el cual lo que se busca estimar es el logaritmo natural de la razón de probabilidades de la ocurrencia de un evento (Wooldridge, 2013).

Y en tal sentido, el modelo logístico es uno dentro de la clase de modelos diseñados para estimar una probabilidad específica de ocurrencia de un evento con respecto a ciertos determinantes que aumentan o disminuyen dicha probabilidad. Este modelo se caracteriza porque al igual que el modelo probit, corrigen los problemas que se generan con un modelo lineal de probabilidad que no garantiza el cumplimiento de ciertas condiciones como la obtención de probabilidades entre cero y uno, la no linealidad de los cambios en probabilidad, la no heteroscedasticidad o la normalidad de los errores (Fuentes, Contreras, y Rodríguez 2018).

\subsection{Incidencias sociales y económicas}

Dentro de las principales incidencias de carácter social que ha traído la pandemia se resaltan elementos propios como el cierre de comercios e industrias, la contracción de la demanda de muchos productos diferentes a los de salud y alimentación, una contracción casi total de turismo a nivel mundial que han generado aumentos del desempleo y una contracción de la economía mundial no vista desde la crisis del 29. Del mismo modo, se viene confirmando como los efectos más profundos los asumen las familias de escasos recursos quienes sienten de manera directa los efectos del desempleo, la falta de ahorros, limitaciones de acceso a adecuados servicios de salud (DANE, 2020).

En el boletín informativo de la situación del mercado laboral en México antes y durante la COVID-19, el Observatorio Internacional de Salarios Dignos (OISAD) da una aproximación con un enfoque de lo que sería el impacto social y económico que generará la COVID-19 para después de la pandemia. Este observatorio hace la comparación de las situaciones previas a la coyuntura mundial, durante la misma y posibles panoramas futuros después de la pandemia. Confirma con base en modelos econométricos, la existencia de precariedad laboral y 
esa es una causa estructural para facilitar la propagación de la COVID-19. Establece, que los vendedores informales son un determinante social que no solo impacta económicamente a las comunidades más vulnerables que requieren subsistir cada día, sino que también inciden en el estado de salud y en especial, en el aumento de la probabilidad de contagio que puedan contraer al estar trabajando en condiciones precarias.

Como estrategia de contención del virus, las cuarentenas han sido una estrategia de aislamiento social que ha servido para frenar la velocidad del contagio y ha dado tiempo a los sistemas de salud para enfrentar la pandemia y reaccionar eficazmente disminuyendo los índices de mortalidad (Ministerio de salud de Colombia, 2020). En este sentido, y con el propósito de verificar el grado de efectividad de la cuarentena, la restricción de la movilidad en los centros de trabajo con reducciones de actividades laborales por encima del $60 \%$ producen tasas más bajas de contagio. El control de la pandemia y la reducción de la movilidad presentan una relación parecida a una $U$ invertida, a medida que se disminuye la movilidad, los contagios siguen creciendo, pasando el umbral del $60 \%$, los contagios empiezan a disminuir, sin embargo, es posible que no en todas partes se logre este nivel dado que una de las principales causas es la estructura económica de mercados informales. (Jiménez B, Peralta, J.; Sánchez, E.; Márquez, I., 2020, p. 4)

\subsection{Patrones socioeconómicos de la COVID -19 en Bogotá}

Para analizar los patrones sociales que pueda tener la incidencia de COVID-19 en poblaciones vulnerables de Bogotá, se tomaron como referencia diferentes artículos y boletines informativos de la facultad de economía de la Universidad de los Andes. En esta serie de publicaciones se muestra la evidencia de un mayor número de contagios en los grupos socioeconómicos más vulnerables de ciudad. Así mismo, se revela que dichas poblaciones son las que más infringen las normas gubernamentales de confinamiento debido a diversos factores económicos intrínsecos de dicho grupo social, como la obligación de subsistencia diaria de su familia. Se evidencia que una persona que vive en estrato bajo resulta 10 veces más probable de ser hospitalizado del mismo modo tiene una probabilidad más alta de fallecer si resulta contagiado y es seis veces más probable ser hospitalizado en una UCI en comparación con una persona de estrato alto.

\section{Figura 1}

Bogotá: afectaciones por COVID por cada 10.000 hogares en el estrato

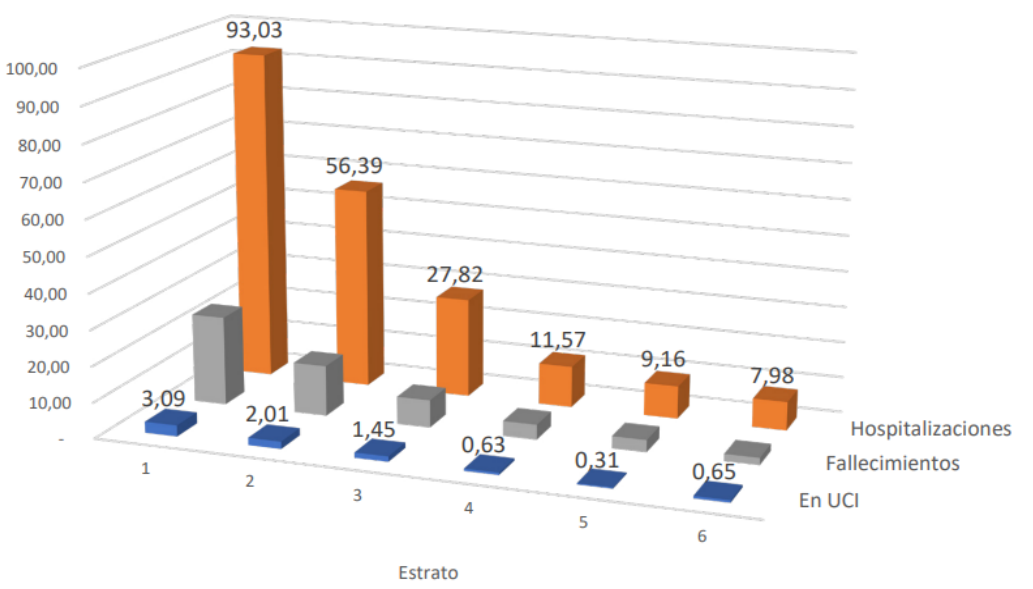

Fuente: Nota Macroeconómica No.23. Grupo de Investigación en Macroeconomía Facultad de Economía Universidad de los Andes. Realizado con datos de SALUDATA, Observatorio de Salud de Bogotá. Fecha de corte 27/07/2020

Siguiendo así, en el estudio se muestra que existe una mayor probabilidad de contagiarse en las poblaciones más vulnerables de estratos socioeconómicos bajos, en comparación con personas de las mismas edades de otros estratos. Dicho estudio plantea una hipótesis, en donde se establece que "es la desigualdad socioeconómica 
misma: las personas más pobres tienen menos probabilidad de aislarse, bien porque no pueden darse el lujo de quedarse en casa o porque prefieren no hacerlo". Del mismo modo se logra evidenciar que dichos estratos y poblaciones son las que albergan mayor cantidad de trabajadores de empresas y los más altos índices de empleo informal. (Grupo de Investigación en Macroeconomía Facultad de Economía Universidad de los Andes, 2020, p. $1,2,3)$

\subsection{Factores sociales ligados a la pobreza}

\section{Desempleo y vulnerabilidad laboral}

En la actualidad debido la situación social y económica, existe una mayor probabilidad de que las personas puedan perder sus empleos, sin embargo, se deben realizar los mayores esfuerzos por preservar los puestos de trabajo y así mismo la estabilidad del poder adquisitivo de las personas, esto permitirá que se estimule la demanda agregada y se dinamice la economía. Para el Observatorio Internacional de Salarios Dignos (OISAD), esta es una recomendación importante para los empresarios, con el fin de que no siga aumentando la tasa de desempleo y con ello que no se incremente la brecha social por los factores sociales ligados a la pobreza. "La Organización Internacional del Trabajo (OIT) estimó que en el primer trimestre del año se paralizaron 130 millones de empleos de tiempo completo y el segundo trimestre serán 305 millones; la región más afectada serían las Américas que perdería hasta una doceava parte de sus plazas laborales." (Jiménez B, Peralta, J.; Sánchez, E.; Márquez, I., 2020, p. 4)

En la misma línea, la Universidad de los Andes de Colombia muestra también incidencia social de COVID-19 por la pérdida de empleos, que se asocia con la hipótesis mencionada de que los estratos más bajos han sido más impactados en la economía de sus hogares, e indica que hay una mayor obligación de movilizarse para satisfacer sus necesidades económicas diarias. Los datos revelan que son las personas con menor nivel de educación las que han enfrentado una mayor probabilidad de perder su empleo en la crisis, en contraste, estas pérdidas de empleo no se concentraban en este grupo en el mismo periodo de 2019. También han sido los que más han visto el impacto de la reducción de empleo en los sectores no esenciales, que son los más limitados por las medidas de confinamiento.

Figura 2

Bogotá: Fracción de desempleados

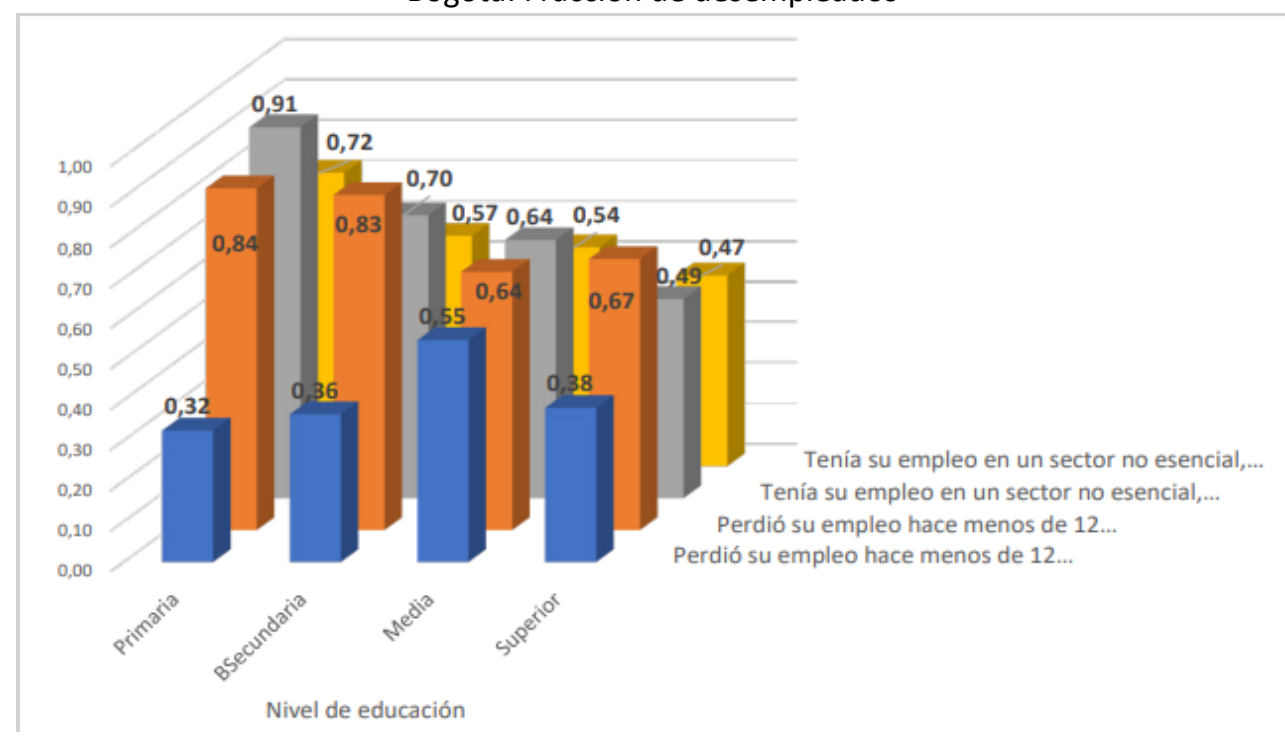

Fuente: Nota Macroeconómica No.23. Grupo de Investigación en Macroeconomía, Facultad de Economía Universidad de los Andes. Realizado con base en datos del GEIH. 


\section{Brechas sociales y causas acumulativas de la pobreza}

En el mundo, una de las principales dificultades sociales es la pobreza, así como todos los factores y variables que esta conlleva. Los países latinoamericanos han sido enormemente afectados a lo largo de su historia por estas problemáticas. Cuando se quiere medir la pobreza no se debe considerar únicamente el nivel de ingresos de las personas, sino que también se reconozca que existe una pobreza multidimensional, en donde se considera como una variable relevante el bajo nivel educativo o las restricciones que existan al momento de acceder a la educación. Es por esto que, para determinar el problema de correlación de la pobreza, se establecen las variables de educación, pobreza y crecimiento económico. En este artículo se menciona que en el trabajo se construye un umbral de pobreza en los países en vías de desarrollo, en donde a partir de un modelo econométrico que incluye variables o factores socioeconómicos, tales como el crecimiento económico, las tasas de corrupción, desempleo y pobreza, para finalmente estimar la probabilidad que tienen los países de incurrir en la llamada «trampa de la pobreza». (Andrade y Jiménez-Bandala, 2018, p. 7,8,17).

En un país que exista un alto nivel de pobreza se obligará a hacer una menor inversión en educación. Cuando se carece de estos niveles de inversiones, conllevan a una deficiente formación profesional, insuficientes niveles en desarrollo en ciencia y tecnología, pobre desarrollo en el nivel productivo, bajo valor agregado en la industria y también conllevaría a no ser atractivo a la inversión extranjera por lo que el crecimiento económico sería lento y esto traería como finalidad altos niveles de pobreza. También se ha comprobado como las trampas de la pobreza tienen una correlación significativa con el desempleo, por tanto, los países en desarrollo requieren de un constante crecimiento en la creación de empleos, que permitan a las personas aumentar sus ingresos y con ello la calidad de vida de sus familias. El empleo tiene una importante incidencia en la capacidad de desarrollo social de los individuos de una región, es por esto que, si existe una disminución de los empleos, la pobreza se prologa por un largo tiempo. (Andrade y Jiménez-Bandala, 2018, p. 7,8,17). Choques externos como los de la pandemia del COVID 19, no solo retrasan la creación de estos empleos sino, que hace que se pierda un camino recorrido para mejorar las condiciones de los sectores más vulnerables.

\subsection{Sector empresarial}

Para poder analizar el impacto que ha generado la COVID-19 en el sector empresarial se deben tener en cuenta las cifras dadas por el Departamento de Estadística de Colombia, en donde para el mes de marzo de 2020 se presentó un decrecimiento de 4,85\% en la economía respecto al mes de marzo de 2019 (DANE, 2010, p.3), lo cual indica una caída económica considerable no vista desde muchos años. Dichos resultados no resultaron alentadores para la economía nacional, sin embargo, se tiene claro que las decisiones que tome el sector empresarial son de vital importancia para contrarrestar la desaceleración económica y la crisis en la que actualmente se presenta.

Como bien se sabe, las economías latinoamericanas tienen como su principal pilar las denominadas Mipymes, es decir, las micro, pequeñas y medianas empresas, que representan un papel clave para contrarrestar la crisis que actualmente se está viviendo causada por la COVID-19. Si se considera que las Mipymes representan más del porcentaje del tejido empresarial en los países latinos y que generan alrededor del 30\% del PIB (BID, 2020), dicho sector empresarial se debe proteger con políticas públicas y ayudas dadas por los gobiernos nacionales, así mismo, los empresarios tienen el desafío de tomar las mejores decisiones para evitar su cierre y con ello, la pérdida de empleos de sus trabajadores. Para la Organización Internacional de Trabajo, se plantean 5 líneas de acción para contrarrestar el efecto económico de la COVID-19 en las Pymes: "1) mayor acceso a financiamiento y a activos circulantes, para garantizar su liquidez en el corto plazo con préstamos o exenciones fiscales temporales; 2) fomento a la demanda de productos y servicios; 3) promoción del empleo y de la protección social, incluyendo subsidios para mantener el empleo de los trabajadores y programas de formación; 4) apoyo a la reapertura de empresas post pandemia; y 5) dialogo social y cohesión social" (Observatorio de la OIT, 2020). 


\section{Metodología y datos}

Para la construcción del modelo se tomaron datos abiertos de la página http://saludata.saludcapital.gov.co/osb/ de la Alcaldía de Bogotá y que es un observatorio de Salud para la ciudad. El archivo de datos se tomó del apartado de los indicadores de enfermedades transmisibles del cual se filtraron el reporte de casos diarios. Con esta información se elaboró una Base de Datos en donde se obtuvo información de personas infectadas para cada una de las localidades de Bogotá, junto con la fecha de los primeros síntomas, la fecha en la que el paciente fue diagnosticado, la edad de la persona, el sexo, el tipo de caso en donde puede tomar valores de en estudio, desconocido, importado y relacionado. Luego de ello se realizó un filtro en el estado del paciente, en donde se tomaron únicamente los casos fallecidos y recuperados. Por último, se tomó el número de datos de vendedores informales por localidad, en la página de datos abiertos de Bogotá para la fecha de 2019. Con los datos obtenidos de la muestra se realizó también una tabla que presenta la cantidad de personas fallecidas de las muestras y el total de recuperados.

Figura 3

Datos de los fallecidos y recuperados por localidad

\begin{tabular}{|l|c|c|l|}
\hline Localidad & Fallecidos (F) & Recuperados (R) & Total \\
\hline Antonio Nariño & 141 & 3396 & 3537 \\
\hline Barrios Unidos & 141 & 4630 & 4771 \\
\hline Bosa & 578 & 22567 & 23145 \\
\hline Chapinero & 138 & 4610 & 4748 \\
\hline Ciudad Bolívar & 574 & 18415 & 18989 \\
\hline Engativá & 581 & 23155 & 23736 \\
\hline Fontibón & 284 & 10114 & 10398 \\
\hline Kennedy & 957 & 32536 & 33493 \\
\hline La candelaria & 41 & 1150 & 1191 \\
\hline Los Mártires & 124 & 3421 & 3545 \\
\hline Puente Aranda & 288 & 9087 & 9375 \\
\hline Rafael Uribe Uribe & 417 & 13530 & 13947 \\
\hline San Cristóbal & 439 & 12213 & 12652 \\
\hline Santa Fe & 134 & 4361 & 4495 \\
\hline Suba & 834 & 30951 & 31785 \\
\hline Teusaquillo & 112 & 3542 & 3654 \\
\hline Tunjuelito & 247 & 6441 & 6688 \\
\hline Usaquén & 379 & 14124 & 14503 \\
\hline Usme & 347 & 10263 & 10610 \\
\hline
\end{tabular}

En la Figura 4 se muestra gráficamente los resultados obtenidos de la muestra. 
Figura 4

Mapas de personas fallecidas vs recuperadas por localidad
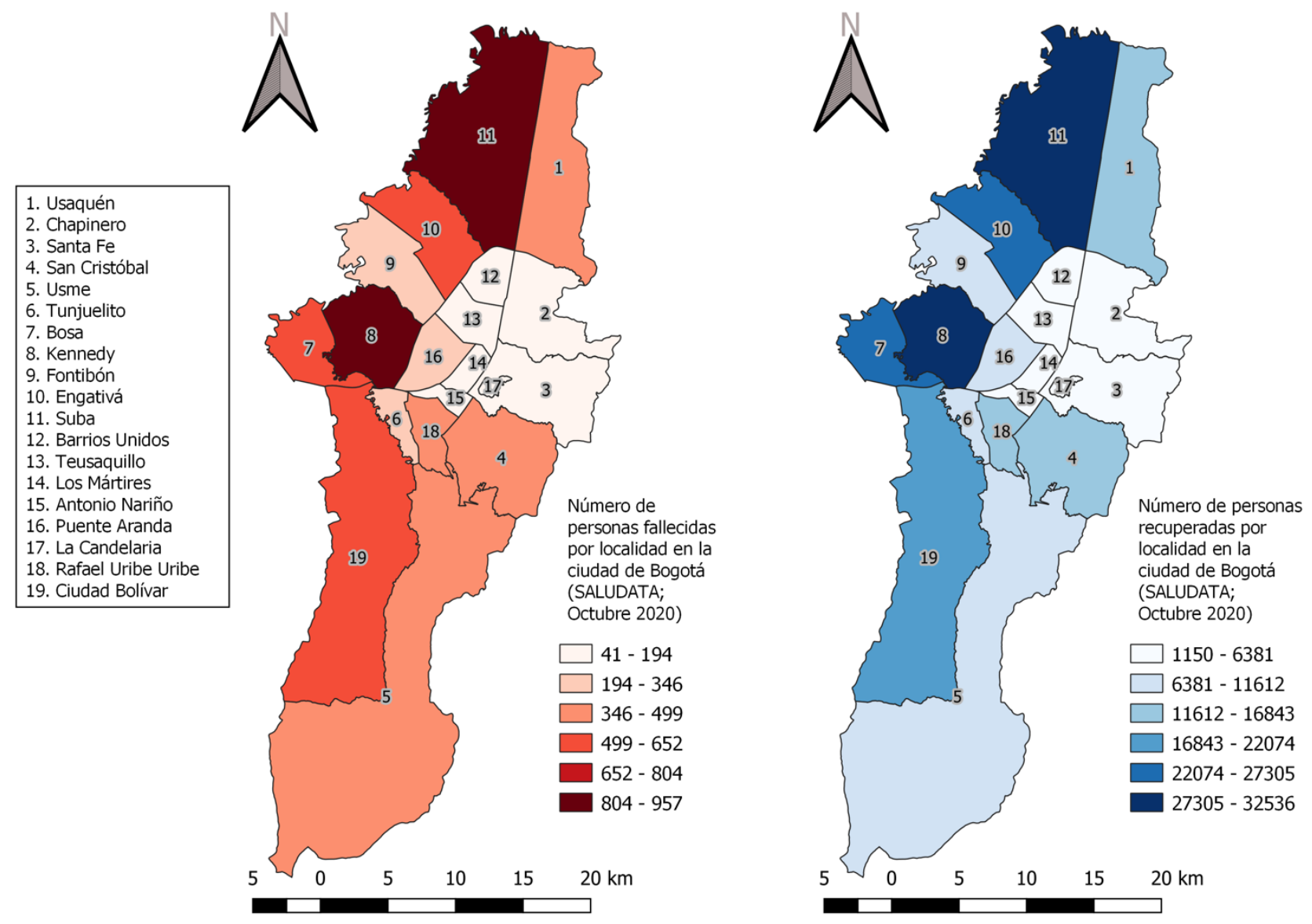

Fuente: Autores a partir de los datos de SALUDATA (2020)

\subsection{Modelo econométrico y resultados}

La siguiente ecuación muestra un modelo que representa la probabilidad de fallecer en función de los determinantes antes considerados, incluyendo el elemento espacial a nivel de localidades, es decir a nivel de división administrativa con ciertas homogeneidades desde un punto de vista geográfico, cultural y económico del distrito capital como lo definió la Ley 768 de 2002.

Fallecido $=\beta_{1}$ edad $+\beta_{2}$ vendedor $+\beta_{3}$ tiempodiag $+\beta_{4}$ hombre $+\beta_{5}$ barriosunidos $+\beta_{6}$ bosa $+\beta_{7}$ chapinero $+\beta_{8}$ ciudadbolivar $+\beta_{9}$ engativa $+\beta_{10}$ fontibon $+\beta_{11}$ kennedy

$+\beta_{12}$ losmartires $+\beta_{13}$ puentearanda $+\beta_{14}$ rafaeluribe $+\beta_{15}$ sancristobal

$+\beta_{16}$ santafe $+\beta_{17}$ suba $+\beta_{18}$ teusaquillo $+\beta_{19}$ tunjuelito $+\beta_{20}$ usaquen $+\beta_{21}$ usme

$+\beta_{22}$ antonionariño $+\beta_{23}$ lacandelaria

Esta ecuación representa la estructura general del modelo de respuesta cualitativa donde:

Fallecido: Es una variable dicótoma donde 1 representa si una persona contagiada de COVID-19 durante los meses de marzo hasta julio en Bogotá D.C, se encuentra fallecida y 0 si se encuentra recuperada.

Edad: Edad en años de la persona infectada. 
Vendedor: Numero de vendedores ambulantes por localidad asociada a cada paciente dependiendo de su ubicación.

Tiempodiag: Tiempo en días transcurrido desde que la persona comenzó a presentar síntomas, hasta que fue diagnosticada con COVID-19.

Hombre: Variable dicótoma que toma el valor de 1 si la persona contagiada es hombre y 0 si es mujer.

Localidad: Variable dicótoma que toma el valor de 1 si la persona pertenece a una localidad especifica (Barrios Unidos, Bosa, Chapinero, Ciudad Bolívar, Engativá, Fontibón, Kennedy, Los Mártires, Puente Aranda, Rafael Uribe Uribe, San Cristóbal, Santa Fe, Suba, Teusaquillo, Tunjuelito, Usaquén, Usme, Antonio Nariño y La Candelaria) y 0 en otro caso.

Para estimar los valores del modelo se recurrió a realizar una serie de regresiones para calcular el logaritmo de la razón de probabilidades, el cual brindó en primera medida, una aproximación de las posibles variables que podrían explicar el fenómeno. La primera decisión que se tomó con base a los resultados, fue la eliminación de las variables de La Candelaria y de Antonio Nariño, debido a que estas, según datos del observatorio, presentan la tasa más baja de contagios en la ciudad y no resultaban muy relevantes en el modelo. Posterior a esto, se volvió a correr la regresión, pero, debido a que la mitad de las variables no resultaron significativas se le aplicó una regresión stepwise la cual se definió una probabilidad específica del 15\% para eliminar las variables que tuvieran los $p$-valores más altos y que mantuviera aquellas que no sobrepasaran el nivel asignado. Al realizar esta operación se eliminaron automáticamente las variables de Teusaquillo y Usme, permitiendo así que la mayoría de variables presentaran una significancia individual.

\section{Resultados}

Debido a que el Modelo Logit solo permite evaluar si la probabilidad del fenómeno estudiado aumente o disminuye según los movimientos de las variables explicativas, se procedió a generar los Odds Ratio de las variables estudiadas. Estos coeficientes "corresponden a un cociente entre dos Odds, siendo un Odds una forma alternativa de expresar la posibilidad de ocurrencia de un evento de interés o de presencia de una exposición" (Cerda et al., 2013), en otras palabras los Odds Ratio representan una razón de probabilidad de la ocurrencia de un evento con respecto de otro. Gracias a este modelo se pudo hacer una primera interpretación de la incidencia de cada variable sobre el fenómeno de estudio.

Figura 5

Resultado de los modelos de respuesta cualitativa

\begin{tabular}{|l|l|l|l|}
\hline Fallecido & Logit & Odds Ratio & \multicolumn{1}{c|}{ dy/dx } \\
\hline Edad & $0.1049208^{* * *}$ & $1.110623^{* * *}$ & $0.0017464^{* * *}$ \\
\hline & 0.001652 & 0.0018348 & 0.00006 \\
\hline Vendedor & $0.0014678^{* * *}$ & $1.001469 * * *$ & $0.0000244^{* * *}$ \\
\hline TiempoDiag & 0.000336 & 0.0003365 & 0.00001 \\
\hline & $-0.0319436 * * *$ & $0.9685612^{* * *}$ & $-0.0005317^{* * *}$ \\
\hline Hombre & 0.0037816 & 0.0036627 & 0.00007 \\
\hline & $0.8744911^{* * *}$ & $2.397655^{* * *}$ & $0.0149216^{* * *}$ \\
\hline Barrios Unidos & 0.0516161 & 0.1237576 & 0.00099 \\
\hline & $1.869544 * * *$ & $6.485335^{* * *}$ & $0.0815727^{* * *}$ \\
\hline Bosa & 0.3585645 & 2.325411 & 0.03126 \\
\hline & -0.1877984 & 0.8287818 & -0.0029128 \\
\hline
\end{tabular}




\begin{tabular}{|c|c|c|c|}
\hline Fallecido & Logit & Odds Ratio & $d y / d x$ \\
\hline \multirow[t]{2}{*}{ Chapinero } & $-2.099693 * * *$ & $0.1224941 * * *$ & $-0.0154474 * * *$ \\
\hline & 0.4802559 & 0.0588285 & 0.00135 \\
\hline \multirow[t]{2}{*}{ Ciudad Bolívar } & $-0.6460909 * *$ & $0.5240905 * *$ & $-0.0084225 * * *$ \\
\hline & 0.2756016 & 0.1444402 & 0.00278 \\
\hline \multirow[t]{2}{*}{ Engativá } & $-1.560175 * * *$ & $0.2100994 * * *$ & $-0.0150022 * * *$ \\
\hline & 0.3841781 & 0.0807156 & 0.00206 \\
\hline \multirow[t]{2}{*}{ Fontibón } & $0.682322 * * *$ & $1.978466 * * *$ & $0.0155411 * *$ \\
\hline & 0.2406313 & 0.4760809 & 0.00727 \\
\hline \multirow[t]{2}{*}{ Kennedy } & $-4.397193 * * *$ & $0.0123119 * * *$ & $-0.0378841 * * *$ \\
\hline & 1.009434 & 0.012428 & 0.00759 \\
\hline \multirow[t]{2}{*}{ Los Mártires } & $-2.495736 * * *$ & $0.0824357 * * *$ & $-0.0163978 * * *$ \\
\hline & 0.6041231 & 0.0498013 & 0.00131 \\
\hline \multirow[t]{2}{*}{ Puente Aranda } & $-1.468007 * * *$ & $0.2303841 * * *$ & $-0.0137029 * * *$ \\
\hline & 0.405026 & 0.0933116 & 0.00195 \\
\hline \multirow[t]{2}{*}{ Rafael Uribe } & $0.7932015^{* * *}$ & $2.210462 * * *$ & $0.0189468 * * *$ \\
\hline & 0.2141509 & 0.4733723 & 0.00706 \\
\hline \multirow[t]{2}{*}{ San Cristobal } & $-2.577541 * * *$ & $0.0759605 * * *$ & $-0.0179766 * * *$ \\
\hline & 0.6186958 & 0.0469965 & 0.00169 \\
\hline \multirow[t]{2}{*}{ Santa Fe } & $-12.21588 * * *$ & $4.95 \mathrm{E}-06 * * *$ & $-0.0220986 * * *$ \\
\hline & 2.833019 & 0.000014 & 0.00164 \\
\hline \multirow[t]{2}{*}{ Suba } & $-1.681218 * * *$ & $0.1861471 * * *$ & $-0.0165086 * * *$ \\
\hline & 0.4193293 & 0.0780569 & 0.00243 \\
\hline \multirow[t]{2}{*}{ Tunjuelito } & $1.620173 * * *$ & $5.053964 * * *$ & $0.0607616 * * *$ \\
\hline & 0.2842284 & 1.43648 & 0.01974 \\
\hline \multirow[t]{2}{*}{ Usaquen } & $1.5504 * * *$ & $4.713355^{* * *}$ & $0.0544075^{* *}$ \\
\hline & 0.4200293 & 1.979747 & 0.02638 \\
\hline \multirow[t]{2}{*}{ Constant } & $-11.03783 * * *$ & & \\
\hline & 0.6235068 & & \\
\hline Observaciones & 35894 & 35894 & 35894 \\
\hline
\end{tabular}

$(*)$ Significancia al $10 \%-(* *)$ Significancia al $5 \%-(* * *)$ Significancia al $1 \%$

Fuente: Autores a partir de los datos de SALUDATA (2020)

De igual manera se muestra en la última regresión los cambios marginales de la probabilidad que corresponde a la última columna de la tabla (dy/dx), es decir que este último si se puede expresar como el cambio en la probabilidad del fenómeno (que la persona contagiada fallezca) cuando aumentan o se cumple cierta condición de la variable explicativa, lo cual facilita su interpretación final al expresar tanto el aumento o la disminución de la probabilidad junto con la proporción de la misma.

A partir de este modelo se obtuvo la regresión final que reúne todos los cambios marginales por variable para la explicación del fenómeno de estudio

Fallecido $=0.0017464$ edad +0.0000244 vendedor -0.000532 tiempodiag +0.0149216 hombre

+0.0815727 barriosunidos -0.015447 chapinero -0.008423 ciudadbolivar

-0.015002 engativa +0.0155411 fontibon -0.037884 kennedy -0.016398 losmartires

-0.013703 puentearanda +0.0189468 rafaeluribe -0.017977 sancristobal

-0.022099 santafe -0.016509 suba +0.0607616 tunjuelito +0.0544075 usaquen 


\section{Análisis de resultados}

Como se pudo evidenciar en el estudio, la mayor parte de las variables propuestas resultaron tener una significancia individual al 1\%, lo cual justifica que las variables espaciales, sociales y económicas tienen una incidencia en la probabilidad que tiene una persona contagiada de COVID-19 de fallecer. Además de ello, como se presenta en el Anexo 1 la prueba de predicción de estat classification realizada por medio de una matriz de confusión, arrojó que el pronóstico del modelo acierta en un $93.55 \%$ de los casos la probabilidad que tienen los contagiados de fallecer. Esta prueba reemplaza el valor del $R^{2}$ ajustado, lo que quiere decir que el modelo presenta una buena bondad en el ajuste donde por cada 100 registros, acierta en aproximadamente 94 de ellos.

Por otra parte, el test de Hosmer-Lemeshow presentado en el Anexo 2, el cual es otro método para estudiar la bondad de ajuste del modelo de regresión logística, consiste en comparar los valores esperados por el modelo con los valores observados. La hipótesis nula del test es que no hay diferencias entre los valores observados y los valores pronosticados, es decir que el rechazo este test indicaría que el modelo tiene un buen ajuste. (Universidad de Santiago de Compostela, 2012). Esta prueba dio como resultado un p valor muy pequeño, lo cual indica que el modelo presenta un buen ajuste, sin embargo, en las pruebas realizadas en los modelos realizados tuvieron como resultado una prueba Prob > chi2 con un p-valor significativo que demuestra que en general el modelo si tiene una significancia global.

Haciendo un análisis individual, se puede encontrar que las probabilidades coinciden, en su mayoría, con la teoría. En el caso de la variable edad, el modelo arrojó que por cada año adicional que tenga la persona, su probabilidad de fallecer, aumenta en un $0.17 \%$, esto coincide con que las personas de la tercera edad son quienes presentan la mayor tasa de mortalidad al contraer el virus SARS-COV-2 causante de la pandemia.

La variable vendedor, que representa la cantidad de vendedores informales por localidad resulto ser significativa y positiva, lo cual también coincide con el hecho de que, entre más vendedores ambulantes haya en una localidad mayor será la probabilidad que tienen los residentes de la misma de fallecer. Esto se relaciona directamente ya que la pandemia ha generado una serie de impactos muy importantes en todos los sectores y la tasa de desempleo para el trimestre móvil febrero - abril de 2020, fue de 14,5\%, superior en 2,6 puntos porcentuales (p.p.), frente a la cifra reportada en el mismo trimestre del año anterior, la cual fue de 11,9\% (Rico, 2020) y ha obligado a muchas personas a recurrir a trabajos informales y buscar otras fuentes de ingresos.

Si bien la enfermedad se ha presentado en una proporción casi que exacta entre hombres y mujeres, estas últimas tienen una ventaja muy por encima del 50,4\% en el tema de contagio, sin embargo, la tasa de mortalidad es mayor en los hombres, siendo en Colombia de 83,1 por cada 100.000 habitantes y en mujeres, 41,3 por cada 100.000 habitantes. Esto se explica debido a que según (Castellanos-Torres et al., 2020) existe "una mayor prevalencia del hábito tabáquico en los hombres, una diferente respuesta inmunitaria o la presencia de otras enfermedades que pueden verse agravadas por la infección (en particular cardiovasculares, respiratorias y diabetes)". En el modelo, la probabilidad de fallecer para los hombres fue positiva y en una proporción de $1.4 \%$, es decir que, si el contagiado de la muestra es hombre, su probabilidad de fallecer es mayor en dicho porcentaje con respecto a las mujeres.

En el caso de las variables espaciales que representan la localidad de residencia de la persona, se obtuvo que la localidad en donde se presenta la tasa más alta de fallecimiento corresponde a Barrios Unidos. En primera instancia este dato puede parecer erróneo debido a que Barrios Unidos se ubica en la posición número $5^{\circ}$ de localidades con la mayor tasa de fallecidos a nivel de la ciudad, sin embargo hay que tener en cuenta de que este ranking es global y no discrimina por el número de personas que residen en dicha localidad, así que bajo este orden de ideas, es lógico que las localidades con los mayores números de habitantes como Kennedy y Suba se ubiquen en los primeros puestos como se mostró en la figura 4. En el estudio realizado se discriminó a cada 
localidad por aparte, es decir que un hallazgo importante es que, a pesar de que la localidad de Barrios Unidos, no tiene una tasa de mortalidad tan alta a nivel de la ciudad, si es la localidad en donde hay mayor cantidad de personas fallecidas por total de recuperados, generando así que en el modelo se presente que la probabilidad de fallecer, si se pertenece a esta localidad aumente en un $8,15 \%$.

\section{Conclusión industria y localidad}

Finalmente se tiene la variable tiempo de diagnóstico, la cual resultó en un hallazgo bastante particular, en donde por cada día que se demore el diagnóstico del paciente, la probabilidad de fallecer disminuye en un $0.0532 \%$. Si bien este resultado es difícil de explicar, el porcentaje no resulta ser tan alto como para concluir que es una variable importante en la probabilidad del fallecimiento del contagiado, sin embargo, esto también se puede deber a que estos tiempos de diagnósticos no son muy precisos (Fernández. L, D, Sanz Gómez, N, Sánchez Serrano, N, Alaoui Sosse, A, \& Aldea -Mansilla, C. 2020, p.1) afirman que "es necesaria la estandarización precisa del diagnóstico hospitalario para reducir el tiempo de respuesta en la confirmación de un caso de sospecha. Por esta razón el diagnóstico de laboratorio es una prioridad de los sistemas de salud pública", es decir que puede que el paciente se le diagnostique la enfermedad muy tarde debido a demoras en las pruebas, o la fecha en la que iniciaron los síntomas no sea muy exacta porque, como bien es sabido, esta enfermedad puede comenzar como una gripe leve a la que le gente podría no darle mucha importancia.

\section{Conclusiones}

Se evidencia que finalmente las poblaciones vulnerables de una sociedad son las más afectadas por este tipo de problemáticas y coyunturas sociales, de esta manera se deben realizar acompañamientos para poblaciones de estratos bajos que no cuenten con los mínimos básicos de supervivencia diaria y de este modo se puedan satisfacer las necesidades que presentan dichas comunidades.

La calidad de vida que tienen las personas es uno de los pilares más importantes en la sociedad y está relacionada con el derecho al trabajo, la disminución del trabajo informal, no obstante, esta el aumento de estos indicadores que ha sido el resultado de las deficientes políticas públicas de los gobiernos de turno. Así pues, al mejorarse la situación laboral del país no solo mejora la economía, sino también se satisfacen de las necesidades básicas de los habitantes, primordialmente para prevenir coyunturas críticas como la que se está presentando actualmente, en donde las personas más vulnerables no tienen opción de acatar la cuarentena obligatoria, debido a la necesidad de sobrevivir diariamente con sus familias. Las personas de bajos estratos y en consecuencia de menores ingresos, caracterizadas por empleos inestables e informales, presentan los mayores problemas sociales de una comunidad, para este caso se evidencian que tienen las tasas más altas de infección y de fallecidos por COVID-19. Se puede evidenciar que las localidades más afectadas son las que presentan un mayor número de habitantes en los estratos socioeconómicos uno y dos.

Bogotá es actualmente la ciudad de Colombia que presenta mayor número de infectados por COVID-19, se evidencia que las poblaciones más vulnerables son las que más problemáticas sociales tienen, aún así se determina que las poblaciones más vulnerables luchan por sobrevivir a las coyunturas socioeconómicas que se presenten. Así mismo, esta población no cuenta con el acceso a un sistema de salud adecuado y depende de la que provee el Estado, pero que refleja una problemática social no solo de la ciudad capital estudiada sino de muchas otras poblaciones de país, tanto en la parte rural como urbana o poblaciones que no tienen ningún tipo de protección social por parte del Estado.

\section{Referencias bibliográficas}

Angulo-Bazán, Y., \& Solis, G. (2020). Factibilidad de las pruebas serológicas en el diagnóstico de COVID-19 en personas fallecidas. Acta Médica Peruana, 37(2), 242-244. 
Castellanos-Torres, E., Tomás Mateos, J., \& Chilet-Rosell, E. (2020). COVID-19 en clave de género. Gaceta Sanitaria. https://doi.org/10.1016/j.gaceta.2020.04.007

Cerda, J., Vera, C., \& Rada, G. (2013). Odds ratio: Aspectos teóricos y prácticos. Revista médica de Chile, 141(10), 1329-1335. https://doi.org/10.4067/S0034-98872013001000014.

DANE. (2020) Informe sobre empleo y brechas de género. Bogotá Colombia. Recuperado de: https://www.dane.gov.co/files/investigaciones/boletines/ech/Informe-sobre-cifras-de-empleo-y-brechasde-genero-10-2020.pdf

Fernández-Lázaro, D., Sanz Gómez, N., Sánchez Serrano, N., Alaoui Sosse, A., \& Aldea -Mansilla, C. (2020). Estandarización de Emergencia para el Diagnóstico del virus SARS-CoV-2 mediante la Reacción en Cadena de la Polimerasa de Transcripción Reversa en Tiempo Real (RT-PCR) en situación de pandemia de COVID19. Revista Madrileña De Salud Pública, 4(7), 1-11. https://doi.org/10.36300/remasp.2020.070.

Fuentes H.J, Amezquita, D. R., \& Murcia, J. C. (2014). Localización de la industria en Bogotá. UD y la GEOMATICA, 9, 75-86. https://doi.org/10.14483/23448407.9186

Fuentes, H. J., Contreras, L. E. C., \& Rodriguez, J. I. (2018). Why Does the Industry Relocate in Colombia? International Journal of Applied Engineering Research, 13(18), 14022-14028.

Gujarati, D. N., \& Porter, D. C. (2010). Econometría (Quinta edición). McGRAW-HILL/INTERAMERICANA EDITORES, S.A.

JIMÉnEZ-BANDALA, C. A., Peralta, J. D., Sánchez, E., Márquez, I., \& Arellano, D. (2020). La situación del mercado laboral en México antes y durante la COVID-19. Revista del Observatorio Internacional de Salarios Dignos, 2(2), 1-9.

Ministerio de Salud de Colombia (2020). En estos tres meses hemos fortalecido una red con capacidad de atención. https://www.minsalud.gov.co/Paginas/En-estos-tres-meses-hemos-fortalecido-una-red-concapacidades-de-atencion.aspx

Nota Macroeconómica No.23. El patrón socioeconómico del COVID. El caso de Bogotá. Grupo de Investigación en Macroeconomía Facultad de Economía Universidad de los Andes, 11 de agosto 2020. Recuperado de: https://economia.uniandes.edu.co/components/com_booklibrary/ebooks/BM\%2023.pdf.

Observatorio de la OIT (2020). La COVID-19 y el mundo del tranajo. Estimaciones actualizadas y análisis. $5^{\circ}$ edición. Ginebra: Oficina Internacional del Trabajo.

Rico, J. A. (2020). Aumenta el desempleo en Bogotá. Observatorio de desarrollo economico. http://observatorio.desarrolloeconomico.gov.co/mercado-laboral-mercado-laboral-general/aumenta-eldesempleo-en-bogota

SALUDATA BOGOTA. (2020), Observatorio de Salud de Bogotá. Fecha de corte 30/07/2020 Recuperado de: http://saludata.saludcapital.gov.co/osb/

Universidad de Santiago de Compostela. (2012). Práctica 6: Regresión Logística I. Departamento de Estadística, Análisis Matemático y Optimización. http://eio.usc.es/eipc1/BASE/BASEMASTER/FORMULARIOS-PHPDPTO/MATERIALES/Mat_50140142_practicaRegLogI_1112.pdf

Wooldridge, J. M. (2009). Introductory econometrics: A modern approach (4th ed). South Western, Cengage Learning.

World Health Organization. Coronavirus disease 2019 (COVID-19) Situation Report - 77 (Internet). 2020 (cited 2020 Abr 6). [ Links ]

Esta obra está bajo una Licencia Creative Commons Attribución-NoCommercial 4.0 International 